\title{
Role of intraband transitions in photocarrier generation
}

\author{
Shunsuke A. Sato, ${ }^{1}$ Matteo Lucchini, ${ }^{2}$ Mikhail Volkov, ${ }^{3}$ Fabian Schlaepfer, ${ }^{3}$ Lukas Gallmann, ${ }^{3}$ \\ Ursula Keller, $^{3}$ and Angel Rubio ${ }^{1,4}$ \\ ${ }^{1}$ Max Planck Institute for the Structure and Dynamics of Matter and Center for Free-Electron Laser Science, \\ Luruper Chaussee 149, 22761 Hamburg, Germany \\ ${ }^{2}$ Department of Physics, Politecnico di Milano, Piazza Leonardo da Vinci 32, 20133 Milano, Italy \\ ${ }^{3}$ Department of Physics, ETH Zürich, 8093 Zürich, Switzerland \\ ${ }^{4}$ Center for Computational Quantum Physics, The Flatiron Institute, 162 Fifth Avenue, New York, New York 10010, USA
}

(Received 10 April 2018; revised manuscript received 4 June 2018; published 5 July 2018)

\begin{abstract}
We theoretically investigate the role of intraband transitions in laser-induced carrier generation for different photon energy regimes: (i) strongly off resonant, (ii) multiphoton resonant, and (iii) resonant conditions. Based on the analysis for the strongly off resonant and multiphoton resonant cases, we find that intraband transitions strongly enhance photocarrier generation in both multiphoton absorption and tunneling excitation regimes, and thus, they are indispensable for describing the nonlinear photocarrier generation processes. Furthermore, we find that intraband transitions enhance photocarrier generation even in the resonant condition, opening additional multiphoton excitation channels once the laser irradiation becomes sufficiently strong. The above findings suggest a potential for efficient control of photocarrier generation via multicolor laser pulses through optimization of the contributions from intraband transitions.
\end{abstract}

DOI: 10.1103/PhysRevB.98.035202

\section{INTRODUCTION}

Thanks to the technological advances in the generation of ultrashort laser pulses over the past few decades, timedomain electron dynamics in atoms and molecules has become accessible [1-5]. Recently, the time-domain observation technique was further applied to solid-state materials, and laserinduced ultrafast electron dynamics in solids was intensively investigated [6-8]. One of the most characteristic features of solid-state systems, compared with atoms and molecules, is the formation of continuum energy bands due to the large number of electrons in the systems. The continuum energy bands enable transitions within each band in addition to transitions among different bands. The first one is called intraband transition, while the latter one is called interband transition. The importance of intraband transitions under intense laser fields has been extensively discussed in the context of high-order-harmonic generation from solids [9-13]. However, their role is still unclear and under debate. Furthermore, it has been demonstrated that intraband processes play a significant role in the ultrafast modification of optical properties of dielectrics under intense laser fields in various conditions $[7,14]$. Recently, the pivotal role of intraband transitions in photocarrier generation of $\mathrm{GaAs}$ was pointed out by Schlaepfer et al. [15].

Photocarrier generation is one of the most fundamental processes in laser-solid interaction as it triggers various phenomena such as band-gap renormalization [16,17], formation of electron-hole plasmas [18], and laser ablation [19,20]. The theoretical description of photocarrier generation under strong fields has been studied for a long time [21-25]. One of the most successful approaches is the Keldysh theory [21], which finds application in many fields [26-28]. The Keldysh theory and its modifications properly capture the effect of intraband transitions and succeed in describing the photocarrier injection rate fairly well. However, the role of intraband transitions in the photocarrier generation process has still remained unclear. Therefore, further understanding of intraband transitions is important for finding a way to enhance or suppress photocarrier generation under rather complex conditions such as complicated materials and multicolor laser fields, which are out of the scope of the Keldysh theory. Recently, the role of intraband transitions in the strong-field resonant regime was investigated, and the strong coupling between Rabi flopping and intraband transitions was predicted for few-cycle pulses [29].

In this work, we theoretically investigate the role of intraband transitions in photocarrier generation with a parabolic two-band model, which is the simplest model to describe semiconductors and insulators. The model has been successfully applied to investigate static as well as dynamical optical properties [30-32]. To explore the carrier-generation processes, we calculate the number of laser-induced carriers with the two-band model in three photon-energy regimes: (i) strongly off resonant, (ii) multiphoton resonant, and (iii) resonant excitation. In each regime, we analyze the effects of intraband transitions on photocarrier generation by artificially suppressing intraband transitions in the two-band model. As a result of the analysis, the impact of intraband transitions will be isolated for each regime.

This paper is organized as follows: In Sec. II we first describe the parabolic two-band model, which will be used in our analysis. Then, we demonstrate the accuracy of the model by comparing it with Kane's band model [33] as well as $a b$ initio simulations. In Sec. III we investigate the role of intraband transitions in photocarrier generation, computing the electron dynamics under laser fields with the two-band model. Finally, our findings are summarized in Sec. IV. 


\section{THEORETICAL MODEL}

In this section, we briefly introduce the parabolic two-band model that will be used in this work. Then, we demonstrate the accuracy of the model for the nonlinear electron dynamics under strong fields by comparing it with the nonparabolic Kane's band model [33] and ab initio calculations based on the time-dependent density functional theory (TDDFT) [34].

\section{A. Parabolic two-band model}

To construct the parabolic two-band model, we start from the following one-body nonrelativistic Schrödinger equation in the dipole approximation:

$$
\begin{aligned}
i \hbar \frac{\partial}{\partial t} u_{b \vec{k}}(\vec{r}, t) & =\left[\frac{1}{2 m_{e}}\left\{\vec{p}+\hbar \vec{k}+\frac{e}{c} \vec{A}(t)\right\}^{2}+v(\vec{r})\right] u_{b \vec{k}}(\vec{r}, t) \\
& =\hat{h}_{\vec{K}(t)} u_{b \vec{k}}(\vec{r}, t),
\end{aligned}
$$

where $m_{e}$ is the electron mass, $u_{b \vec{k}}(\vec{r}, t)$ is a time-dependent Bloch state, and $v(\vec{r})$ is a one-body potential that has the same periodicity as the crystal. Here, $b$ denotes a band index, and $\vec{k}$ denotes the Bloch wave number. For the two-band model, we do not explicitly define an analytical form of the one-body potential $v(\vec{r})$. Instead, we will directly define eigenvalues and matrix elements of the eigenstates of the Hamiltonian in Eq. (1). We note that the crystal momentum is shifted by the vector potential as $\vec{K}(t)=\vec{k}+e \vec{A}(t) / \hbar c$ based on the acceleration theorem.

Then, we introduce the Houston state $[35,36]$ as

$$
u_{b \vec{k}}^{H}(\vec{r}, t)=\exp \left[-\frac{i}{\hbar} \int^{t} d t^{\prime} \epsilon_{b \vec{K}\left(t^{\prime}\right)}\right] u_{b \vec{K}(t)}^{S}(\vec{r}),
$$

where $\epsilon_{b \vec{K}(t)}$ and $u_{b \vec{K}(t)}^{S}(\vec{r})$ are an eigenvalue and the eigenstate of the instantaneous Hamiltonian $\hat{h}_{\vec{K}(t)}$, respectively;

$$
\hat{h}_{\vec{K}(t)} u_{b \vec{K}(t)}^{S}(\vec{r})=\epsilon_{b \vec{K}(t)} u_{b \vec{K}(t)}^{S}(\vec{r}) .
$$

To construct a two-band model, we assume that the wave function at each $k$ point can be expanded by only two Houston states: one representing a valence and the other representing a conduction state:

$$
u_{\vec{k}}(\vec{r}, t)=c_{v \vec{k}}(t) u_{v \vec{k}}^{H}(\vec{r}, t)+c_{c \vec{k}}(t) u_{c \vec{k}}^{H}(\vec{r}, t) .
$$

Inserting Eq. (4) into Eq. (1), we can derive an equation of motion for the coefficients $c_{v \vec{k}}(t)$ and $c_{c \vec{k}}(t)$,

$$
i \hbar \frac{d}{d t}\left(\begin{array}{l}
c_{v \vec{k}}(t) \\
c_{c \vec{k}}(t)
\end{array}\right)=\left(\begin{array}{cc}
0 & h_{v c, \vec{k}}(t) \\
h_{v c, \vec{k}}^{*}(t) & 0
\end{array}\right)\left(\begin{array}{l}
c_{v \vec{k}}(t) \\
c_{c \vec{k}}(t)
\end{array}\right),
$$

where the off-diagonal matrix element is given by

$$
h_{v c, \vec{k}}(t)=-\frac{i \vec{p}_{v c, \vec{K}(t)} \cdot \vec{E}(t)}{\epsilon_{v, \vec{K}(t)}-\epsilon_{c, \vec{K}(t)}} \frac{e \hbar}{m} e^{\frac{1}{i \hbar} \int^{t} d t^{\prime}\left\{\epsilon_{c, \vec{K}\left(t^{\prime}\right)}-\epsilon_{v, \vec{K}\left(t^{\prime}\right)}\right\}}
$$

and

$$
\vec{p}_{v c, \vec{K}(t)}=\int_{\Omega} d \vec{r} u_{v \vec{K}(t)}^{S, *}(\vec{r}) \vec{p} u_{c \vec{K}(t)}^{S}(\vec{r}),
$$

where $\Omega$ is the volume of the unit cell. Note that Eq. (5) is nothing but the Houston state expansion of the Schrödinger equation $[35,36]$ with only two Houston states.
To further simplify the model, we apply two approximations: (i) a parabolic band approximation and (ii) a uniform matrix-element approximation. The parabolic band approximation allows us to describe the electronic structure by the following quadratic forms:

$$
\begin{aligned}
\epsilon_{v, \vec{k}} & =-\frac{\hbar^{2} \vec{k}^{2}}{2 m_{v}}, \\
\epsilon_{c, \vec{k}} & =\epsilon_{g}+\frac{\hbar^{2} \vec{k}^{2}}{2 m_{c}},
\end{aligned}
$$

where $\epsilon_{g}$ is the band gap and $m_{v}$ and $m_{c}$ are the effective masses for valence and conduction bands, respectively. The uniform matrix-element approximation allows us to ignore the $k$ dependence of the matrix element,

$$
\vec{p}_{v c, \vec{K}(t)}=\vec{p}_{v c}
$$

Note that the uniformity of the matrix elements has been confirmed by $a b$ initio simulations for several semiconductors [37].

The derived parabolic two-band model is the simplest model for semiconductors and insulators. The accuracy of this model in the context of carrier generation under strong fields will be evaluated in the following section.

\section{B. Comparison with other models}

To demonstrate the accuracy of the parabolic two-band model, we compare it with the nonparabolic Kane's band model [33] and first-principles calculations based on the TDDFT.

In this work, we consider the laser-induced electronic excitation in $\alpha$-quartz. To describe $\alpha$-quartz, we set the effective mass $m_{r}=1 /\left(m_{v}^{-1}+m_{c}^{-1}\right)$ to $0.4 m_{e}$ and the band gap $\epsilon_{g}$ to $9.0 \mathrm{eV}$ according to Ref. [38]. The transition momentum matrix $p_{v c}$ is evaluated by Kane's formula [39],

$$
p_{v c}=\frac{1}{2} \sqrt{\frac{\epsilon_{g}}{m_{r}}} .
$$

The laser-induced electronic excitation energy $E_{e x}$ for the twoband model is given by

$$
E_{e x}=\frac{2 n_{c}}{(2 \pi)^{3}} \int d \vec{k}\left(\epsilon_{c \vec{k}}-\epsilon_{v \vec{k}}\right)\left|c_{c \vec{k}}\left(t_{f}\right)\right|^{2},
$$

where $t_{f}$ is the time at which the laser irradiation ends. Here, we introduced a dimensionless factor $n_{c}$ so that the two-band model reproduces the first-principles calculation (see Fig. 1). Since the electronic structure of the material is approximated by only the two bands, the effective electron density that contributes to the response may not be well described. Thus, the factor $n_{c}$ can be understood as a correction for the effective electron density. In this work, we set $n_{c}$ to 20 .

For the nonparabolic band model, we employ the following Kane's band [33] instead of the parabolic band:

$$
\epsilon_{c \vec{k}}-\epsilon_{v \vec{k}}=\epsilon_{g}\left(1+\frac{\hbar^{2} k^{2}}{m_{r} \epsilon_{g}}\right)^{1 / 2} .
$$

We note that the Keldysh formula is derived based on this Kane's band model [21]. 


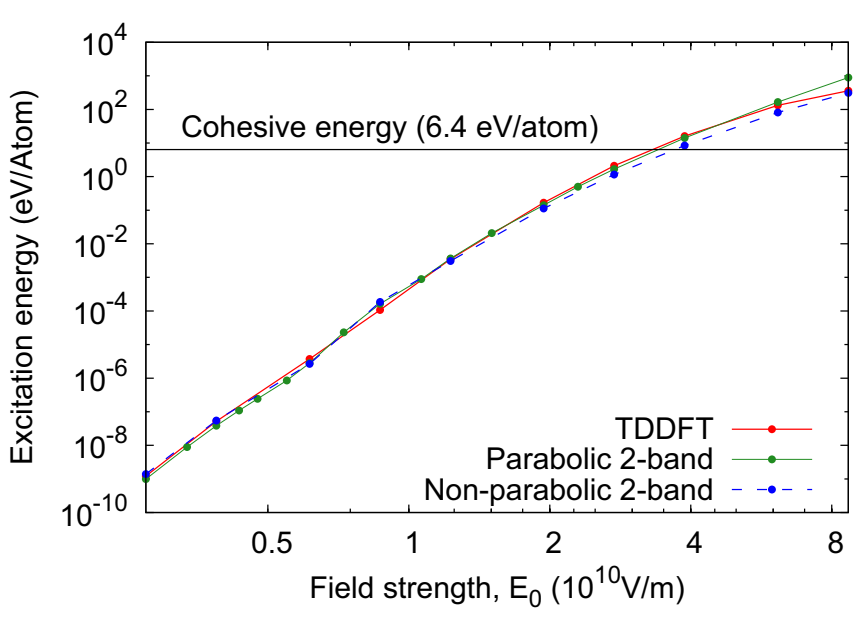

FIG. 1. Laser-induced electronic excitation energy in $\alpha$-quartz after the laser irradiation. The dots represent the results of the TDDFT calculation (red dots and solid line), the parabolic two-band model (green dots and solid line), and the nonparabolic two-band model (blue dots and dashed line).

For the ab initio modeling, we employ real-time real-space TDDFT calculations with a norm-conserving pseudopotential [40]. For the exchange-correlation potentials, the BeckeJohnson exchange [41] and Perdew-Wang correlation [42] are employed. For practical simulations, we employed a TDDFT code, the $a b$ initio real-time electron dynamics simulator $[43,44]$. Technical details of the real-time, real-space TDDFT calculations are explained elsewhere [45,46]. Here, we describe only the numerical parameters for the TDDFT calculation in this work: we employ a rectangular unit cell which contains 6 silicon atoms and 12 oxygen atoms. The unit cell is discretized into a Cartesian $20 \times 36 \times 50$ grid. For the Brillouin zone sampling, we employ $4^{3} k$ points.

In this work, the applied electric field is described by the following vector potential:

$$
\vec{A}(t)=-\vec{e}_{c} \frac{E_{0}}{\omega} \sin ^{4}\left(\pi \frac{t-\frac{T}{2}}{T}\right) \sin \left[\omega\left(t-\frac{T}{2}\right)\right]
$$

in the domain $0<t<T$ and zero outside of it. Here, $\vec{e}_{c}$ is the unit vector for the $c$ axis of $\alpha$-quartz, $E_{0}$ is the maximum electric field strength, $T$ is the full pulse duration, and $\omega$ is the mean frequency.

Here, we set the mean frequency $\omega$ to $1.55 \mathrm{eV} / \hbar$ and the full duration $T$ to $30 \mathrm{fs}$. We note that the corresponding full width at half maximum duration of the laser intensity profile is about $7.8 \mathrm{fs}$. By changing the maximum field strength $E_{0}$, we compute the laser-induced electronic excitation energy.

Figure 1 shows the laser-induced electronic excitation energy calculated with TDDFT (red), the parabolic two-band model (green), and the nonparabolic two-band model (blue). The black horizontal line indicates the cohesive energy of $\alpha$-quartz, $6.4 \mathrm{eV} /$ atom [47], which is regarded as a reference for the laser-ablation threshold [48]. We can see that the parabolic two-band model provides almost the same result as the nonparabolic Kane's band model. This fact indicates that the anharmonicity of the band structure does not have

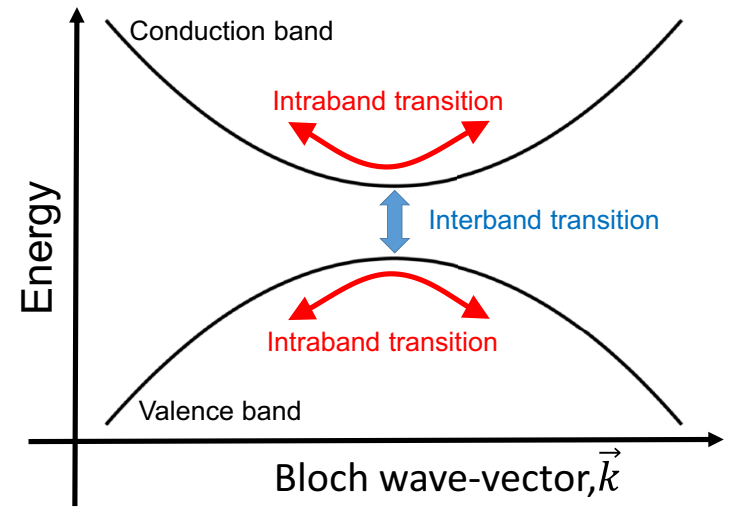

FIG. 2. Schematic picture of intraband transitions (red) and interband transitions (blue) in the parabolic two-band model.

significant effects on photocarrier generation in the present conditions.

Furthermore, we emphasize that both the parabolic and nonparabolic two-band models show reasonable agreement with the first-principles results for the full intensity range studied here. Therefore, the accuracy of this simplified parabolic two-band model for the analysis of the laser-induced electronic excitation is demonstrated. Note that the real-time, real-space TDDFT calculation adequately captures the full band structure of solids and well describes the motion of electrons in the Brillouin zone, including Bloch oscillations. Therefore, the agreement between the two-band model and the TDDFT calculation indicates a low significance of the Bloch oscillation for photocarrier generation in the present regime.

We note that, while the parabolic two-band model shows nice agreement with the TDDFT calculation in the strongly off resonant condition, it may show deviations in other conditions due to a multiband effect. Although the multiband effect is important to describe realistic systems, we completely omit it in the present work in order to clearly understand the role of intraband transitions. The multiband effect and its coupling with intraband transitions will be investigated in future work based on this work.

\section{EFFECTS OF INTRABAND TRANSITIONS}

In this section, we investigate the impact of intraband transitions on photocarrier generation, employing the parabolic two-band model described in the previous section. Figure 2 shows a schematic picture of intraband and interband transitions; intraband transitions correspond to the horizontal motion in the $k$ space, while interband transitions correspond to the vertical transition.

In the two-band model, intraband transitions are described by the shift of the crystal momentum $\vec{K}(t)$ in the instantaneous eigenstates in Eq. (4), while interband transitions are described by transitions between the valence and the conduction Houston states via the off-diagonal elements of the Hamiltonian matrix. Therefore, we can omit intraband transitions from the model by neglecting the crystal momentum shift due to the vector potential: $\vec{K}(t)=\vec{k}+e \vec{A}(t) / \hbar c \rightarrow \vec{k}$. In order to investigate the impact of intraband transitions, we compare the parabolic two-band model with (i) both intra- and interband transitions 


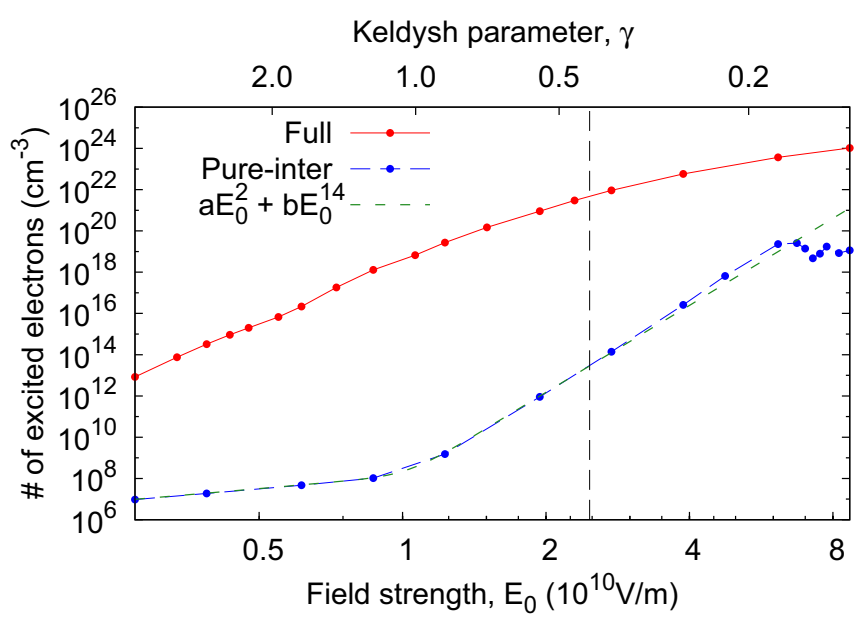

FIG. 3. Number of excited electron-hole pairs after the laser irradiation in the strongly off resonant regime, computed using Eq. (16). The dots represent the result of the full model (red dots and solid line), and the pure interband model (blue dots and dashed line). A polynomial fitting by $a E_{0}^{2}+b E_{0}^{14}$ is also shown as a green short-dashed line.

enabled and (ii) only interband transitions. We call the first one the full model and the latter the pure interband model. A similar analysis has been done by Golde et al. for high-order-harmonic generation in a semiconductor using the semiconductor Bloch equations [49].

\section{A. Strongly off resonant condition: $\hbar \omega / \epsilon_{g} \sim 0.17$}

First, we investigate the effect of intraband transitions in the strongly off resonant regime, where the mean photon energy, $\hbar \omega=1.55 \mathrm{eV}$, is much smaller than the band gap, $\epsilon_{g}=9 \mathrm{eV}$. The full pulse duration is set to $T=30 \mathrm{fs}$. The corresponding bandwidth of the laser pulse is about $0.5 \mathrm{eV}$. It is well known that photocarrier generation in such a strongly off resonant regime is well described by Keldysh's formula [21] with the so-called Keldysh parameter,

$$
\gamma=\frac{\omega \sqrt{m_{r} \epsilon_{g}}}{e E_{0}}=\frac{1}{2} \sqrt{\frac{\epsilon_{g}}{U_{p}}},
$$

where $U_{p}$ is the ponderomotive energy, $U_{p}=e^{2} E_{0}^{2} / 4 m_{r} \omega^{2}$. The Keldysh parameter is commonly used to determine the excitation regime [50]: a large value $(\gamma \gg 1)$ indicates that multiphoton absorption dominates the excitation mechanism, while a small value $(\gamma<0.5)$ indicates that the tunnel mechanism dominates. We may expect that intraband transitions play a significant role in photocarrier generation in the strongly off resonant regime since the ponderomotive energy $U_{p}$, which originates from the quiver motion of the crystal momentum $\vec{K}(t)$ due to intraband transitions, is linked to the excitation rate through $\gamma$ in the Keldysh theory [21].

Figure 3 shows the number of excited electrons after laser irradiation, computed by the following formula:

$$
n_{e x}=\frac{2 n_{c}}{(2 \pi)^{3}} \int d \vec{k}\left|c_{c \vec{k}}\left(t_{f}\right)\right|^{2} .
$$

This expression corresponds to the population in the conduction band computed by the projection onto the conduction Houston states $u_{c \vec{k}}^{H}(\vec{r}, t)$.

In Fig. 3, the red points show the result of the full model, and the blue points show that of the pure interband model. As expected, we see that photocarrier generation is significantly affected by the omission of intraband transitions in the whole investigated intensity region, and as a consequence, photocarrier generation is strongly suppressed in the pure interband case.

Here, we find that the number of photoexcited carriers of the pure interband model can be well described by a simple polynomial as a function of the field strength $n_{e x}\left(E_{0}\right)=\alpha E_{0}^{2}+\beta E_{0}^{14}$ even in the high-intensity region. The first term corresponds to photocarrier generation by single-photon absorption due to the high-energy tail of the pulse spectrum, while the second term corresponds to the absorption of seven photons. Note that, since even-number multiphoton absorption processes are forbidden in the pure interband model, the seven-photon absorption process is the lowest-order nonlinear excitation process even though the energy of six photons exceeds the gap.

This fact indicates that photocarrier generation without intraband transitions is dominated by the multiphoton absorption process even at high intensities, where the Keldysh parameter $\gamma$ is of the order of 1 or less. Note that the Keldysh parameter $\gamma$ becomes 1 when the maximum field $E_{0}$ is about $10^{10} \mathrm{~V} / \mathrm{m}$ in the present conditions (see the secondary $x$ axis in Fig. 3). We can understand the predominance of the multiphoton absorption process in the pure interband model based on the ponderomotive energy. As mentioned above, the ponderomotive energy originates from the quiver motion due to intraband motion. Thus, the omission of intraband transitions corresponds to setting the ponderomotive energy $U_{p}$ to zero, and the Keldysh parameter $\gamma$ becomes infinity. Therefore, we expect the excitation process to be dominated by multiphoton absorption once intraband transitions are neglected.

To obtain further insight into the role of intraband transitions, we calculate the energy distribution of electron-hole $(e-\mathrm{h})$ pairs at each $e$-h excitation energy $n_{e-h}\left(E_{e-h}\right)$. Since we consider the two-band model in this work, the distribution of $e$-h pairs at each $e$-h excitation energy $E_{e-h}$ can be evaluated by

$$
n_{e-h}\left(E_{e-h}\right)=\frac{2 n_{c}}{(2 \pi)^{3}} \int d \vec{k}\left|c_{c \vec{k}}\left(t_{f}\right)\right|^{2} \delta\left(\epsilon_{c \vec{k}}-\epsilon_{v \vec{k}}-E_{e-h}\right) .
$$

Figure 4 shows the distribution of $e$-h pairs as a function of the $e$-h energy after laser irradiation with a field strength of $E_{0}=2.74 \times 10^{10} \mathrm{~V} / \mathrm{m}$, which is marked with the black dashed vertical line in Fig. 3. The red solid line shows the result of the full model, while the blue dashed line shows that of the pure interband model. We see that the $e$-h distribution of the pure interband model shows a single peak. As discussed above, the carrier injection in the pure interband model at the present field strength is dominated by the seven-photon absorption process. This is also reflected in the position of the single peak of the pure interband model, which corresponds to the energy of the seven absorbed photons $(1.55 \mathrm{eV} \times 7=$ $10.85 \mathrm{eV})$. In contrast, the $e-\mathrm{h}$ distribution of the full model 


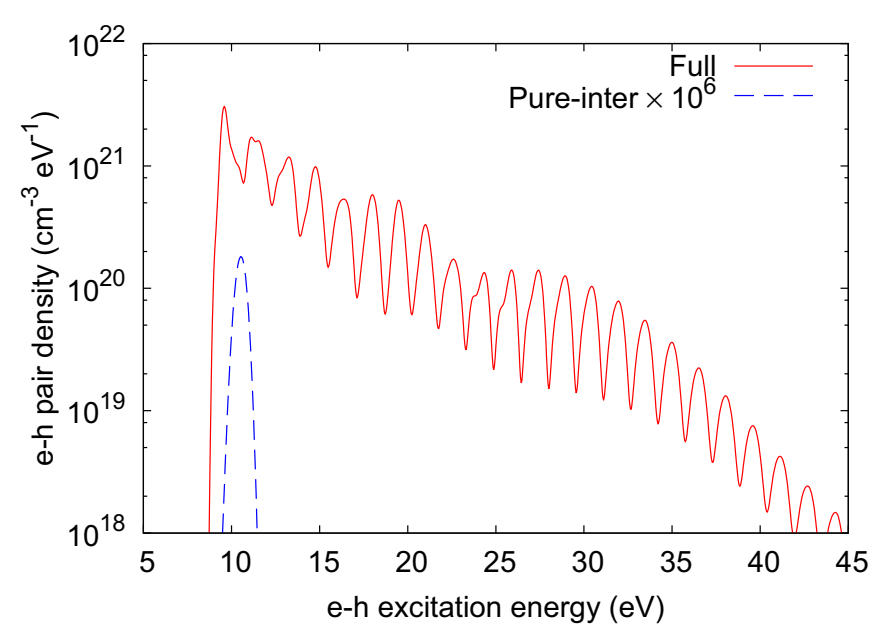

FIG. 4. Distribution of photoinduced electron-hole pairs in the strongly off resonant condition $(\hbar \omega=1.55 \mathrm{eV})$ with the field strength of $2.74 \times 10^{10} \mathrm{~V} / \mathrm{m}$. The results of the full model (red solid line) and the pure interband model (blue dashed line) are shown.

shows multiple peaks that are distributed over a broad energy range. Furthermore, we may see a plateau around $25-30 \mathrm{eV}$. Such a multipeak structure with a plateau region can also be found in above-threshold-ionization (ATI) photoelectron spectra of atoms [51-53]. The plateau structure of the ATI spectra is formed by the scattering of ionized electrons from parent ions under laser fields [54]. This indicates that the plateau structure in the $e$-h distribution of Fig. 4 is also formed by some scattering process driven by the strong electric fields.

The cutoff energy of the plateau structure of the ATI spectra and the high-order-harmonic generation from atoms are well understood based on semiclassical models [54-56]. Recently, the semiclassical model for high-order-harmonic generation was extended to solid-state systems [57]. Likewise, constructing a classical analog to correctly understand the plateau structure in Fig. 4 as well as to develop an intuitive description of photocarrier injection under strong fields is important. However, since a detailed analysis of the plateau structure based on a semiclassical treatment is beyond the scope of the present work, this aspect will be investigated in future work.

We note that the detailed structure of the $e$-h distribution must be immediately smeared out by electron-electron, electron-phonon, and electron-impurity scattering processes in a realistic system. Therefore, it would be hard to experimentally observe the $e$-h distribution shown in Fig. 4 .

\section{B. Multiphoton resonant condition: $\hbar \omega / \epsilon_{g}=1 / 3$}

Next, we investigate the effect of intraband transitions in the multiphoton resonant condition. For this purpose, we set the mean photon energy of the laser pulse $\hbar \omega$ to $3 \mathrm{eV}$, which is identical to one third of the band gap $\epsilon_{g}$. As a result, resonant excitation is obtained by absorbing three photons. The full pulse duration is set to $T=30 \mathrm{fs}$.

Figure 5 shows the injected carrier population as a function of the laser field strength $E_{0}$ in the three-photon resonant condition. The sixth power of the field strength $E_{0}^{6}$ is also

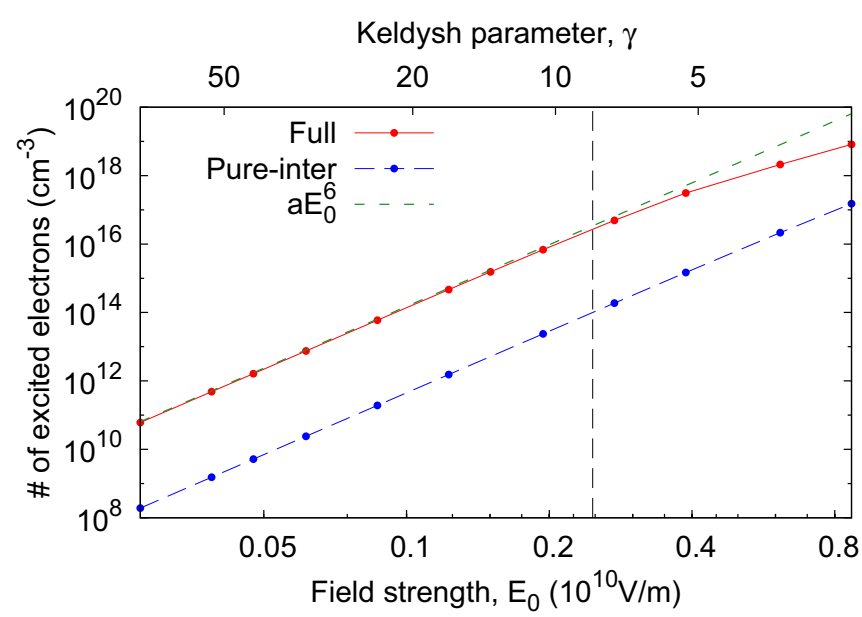

FIG. 5. Number of excited electron-hole pairs after the laser irradiation in the three-photon resonant regime. The dots represent the result of the full model (red dots and solid line) and the pure interband model (blue dots and dashed line). A polynomial fitting by $a E_{0}^{6}$ is also shown as a green short-dashed line.

shown by a green short-dashed line. As seen from Fig. 5, the population of both the full model and the pure interband model is proportional to the sixth power of the field strength in the weak-field region, indicating that photocarrier injection is dominated by three-photon absorption. Although the threephoton absorption process dominates the carrier injection in both models, the full model shows an about 300 times higher injected population than the pure interband model. This fact means that intraband transitions strongly enhance the multiphoton excitation process.

Since intraband transitions cannot directly inject any carriers by themselves, the enhancement can be understood as photoassisted carrier injection via additional excitation paths opened by intraband transitions. Note that if intraband transitions are induced by a static electric field and interband transitions are induced by an optical field, carrier injection by optical absorption corresponds to photoassisted tunneling, which is also known as the Franz-Keldysh effect [58,59].

Figure 6 shows the distribution of $e$-h pairs after laser irradiation with a field strength of $E_{0}=0.274 \times 10^{10} \mathrm{~V} / \mathrm{m}$, which is marked by the black dashed vertical line in Fig. 5 . The red solid line shows the result of the full model, while the blue dashed line shows that of the pure interband model. As seen from Fig. 6, the first peak at around $9 \mathrm{eV}$ dominates the total population in both models. This fact is consistent with the above finding that the photocarrier injection is dominated by the three-photon absorption process. Furthermore, the first peak is strongly enhanced by intraband transitions. Therefore, we can clearly conclude that intraband transitions strongly assist the lowest-order multiphoton absorption process and largely enhance photocarrier injection.

In Fig. 6, we see that intraband transitions induce additional peaks in the higher-energy region. For the present field strength $E_{0}=0.274 \times 10^{10} \mathrm{~V} / \mathrm{m}$, contributions from these additional peaks are rather small compared with the primary peak at around $9 \mathrm{eV}$. However, once the field strength becomes large enough and the corresponding Keldysh parameter becomes 


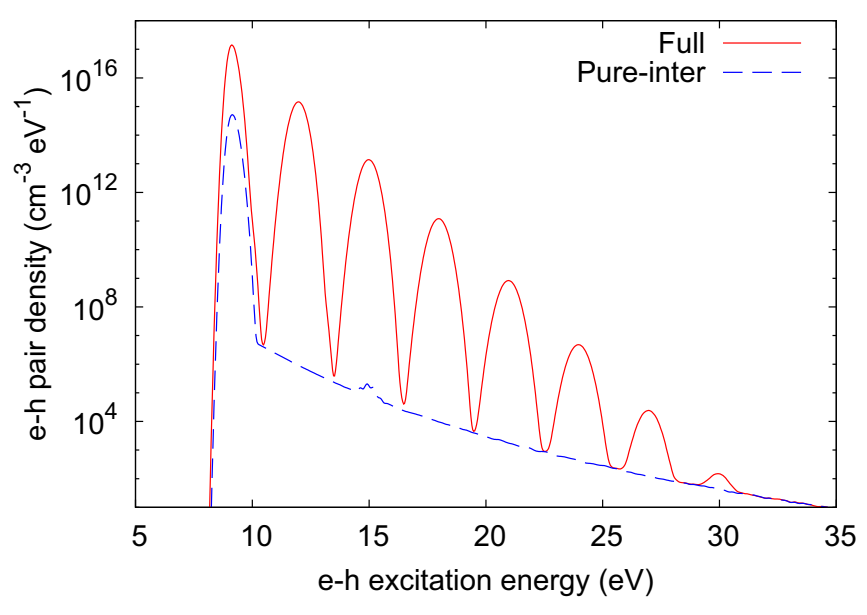

FIG. 6. Distribution of photoinduced electron-hole pairs in the three-photon resonant condition with a field strength of $0.274 \times$ $10^{10} \mathrm{~V} / \mathrm{m}$. The results of the full model (red solid line) and the pure interband model (blue dashed line) are shown.

small enough, these additional peaks are important, as discussed in Sec. III A.

To obtain further insight into the carrier-injection enhancement by intraband transitions, we analytically study photocarrier generation based on perturbation theory. For simplicity, we consider the carrier injection only at the $\Gamma$ point $(k=0)$, assuming that the laser pulse has a rectangular envelope with duration $T$. Based on the third-order perturbation theory, the injected population of the pure interband model is given by

$$
n_{\Gamma}^{\text {pure-inter }}=\left|\frac{1}{8}\left(\frac{\vec{p}_{v c} \cdot \vec{E}_{0}}{\epsilon_{g}} \frac{e \hbar}{m}\right) \frac{15}{16} \frac{1}{\hbar^{2} \omega} \Delta_{S} T\right|^{2},
$$

where $\Delta_{S}$ is the cycle-averaged Stark shift induced by interband transitions,

$$
\Delta_{S}=\left(\frac{\vec{p}_{v c} \cdot \vec{E}_{0}}{\epsilon_{g}} \frac{e \hbar}{m}\right)^{2} \frac{1}{\epsilon_{g}} .
$$

In the full model, intraband transitions open an additional excitation path. The injected population via the additional path can be evaluated by

$$
n_{\Gamma}^{\text {intra-assisted }}=\left|\frac{1}{8}\left(\frac{\vec{p}_{v c} \cdot \vec{E}_{0}}{\epsilon_{g}} \frac{e \hbar}{m}\right) \frac{1}{\hbar^{2} \omega} U_{p} T\right|^{2} .
$$

A detailed derivation of Eqs. (18) and (20) will be described in Appendix A.

With the present parametrization, the injected carrier population via the additional path $n_{\Gamma}^{\text {intra-assisted is about } 100 \text { times }}$ larger than that of the pure interband path $n^{\text {pure-inter }}$. This fact is consistent with the above enhancement by intraband transitions in the numerical simulations.

Comparing Eqs. (18) and (20), we see that the ratio of the injected population via two different excitation paths can be well approximated by the square of the ratio of the ponderomotive energy and the Stark shift:

$$
n_{\Gamma}^{\text {intra-assisted }} / n_{\Gamma}^{\text {pure-inter }} \approx\left|U_{p} / \Delta_{S}\right|^{2} .
$$

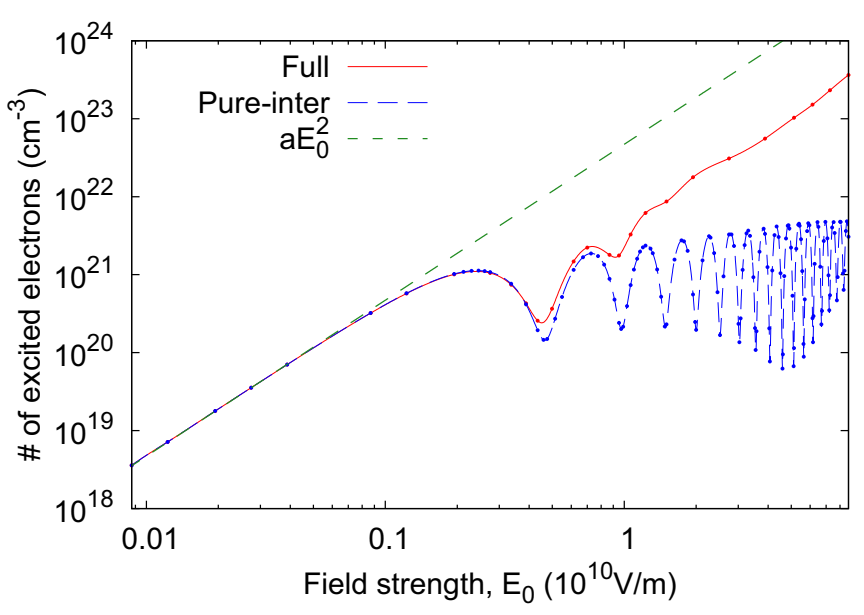

FIG. 7. Number of excited electron-hole pairs after the laser irradiation in the resonant regime. The dots represent the result of the full model (red dots and solid line), and the pure interband model (blue dots and dashed line). The square of the field strength, $E_{0}^{2}$, is also shown as the green short-dashed line.

Since only the ponderomotive energy depends on the photon energy of laser fields, Eq. (21) indicates that the additional excitation path, which is opened by intraband transitions, becomes significant in the strongly off resonant regime, where the photon energy becomes small. Furthermore, in higherorder nonlinear responses, the higher-order ratio $\left(U_{p} / \Delta_{S}\right)^{n}$ is expected to be a characteristic parameter instead of Eq. (21). Therefore, once the ponderomotive energy becomes substantially large, the higher-order nonlinear responses can be strongly suppressed by the omission of intraband transitions. In fact, these expectations are consistent with the above findings from the numerical simulations: the omission of intraband transitions strongly suppresses photocarrier generation in the off-resonant condition, as seen from Fig. 3. Moreover, the higher-order multiphoton peaks are strongly suppressed by the omission of intraband transitions, as seen from Figs. 4 and 6.

Combining the findings in this section with those in Sec. III A, we can conclude that intraband transitions play a crucial role in nonlinear photocarrier injection in both multiphoton and tunneling regimes.

\section{Resonant condition: $\hbar \omega / \epsilon_{g}=1$}

Finally, we investigate the effect of intraband transitions in the resonant case. For the sake of the investigation, we set the mean photon energy of the laser pulse $\hbar \omega$ to $9 \mathrm{eV}$, which is identical to the band gap $\epsilon_{g}$. Therefore, single-photon absorption dominates the carrier generation in the weak-field regime. The full pulse duration is set to $T=30 \mathrm{fs}$.

Figure 7 shows the carrier population as a function of the laser field strength. We see that the result of the full model coincides with that of the pure interband model in the weak-field region. This fact means that intraband transitions do not play any role in the weak-field resonant condition. Around a field strength of $0.3 \times 10^{10} \mathrm{~V} / \mathrm{m}$, the number of excited electrons decreases as the intensity increases. This feature indicates depopulation of excited electrons through the onset of Rabi flopping. In the high-intensity region, the 


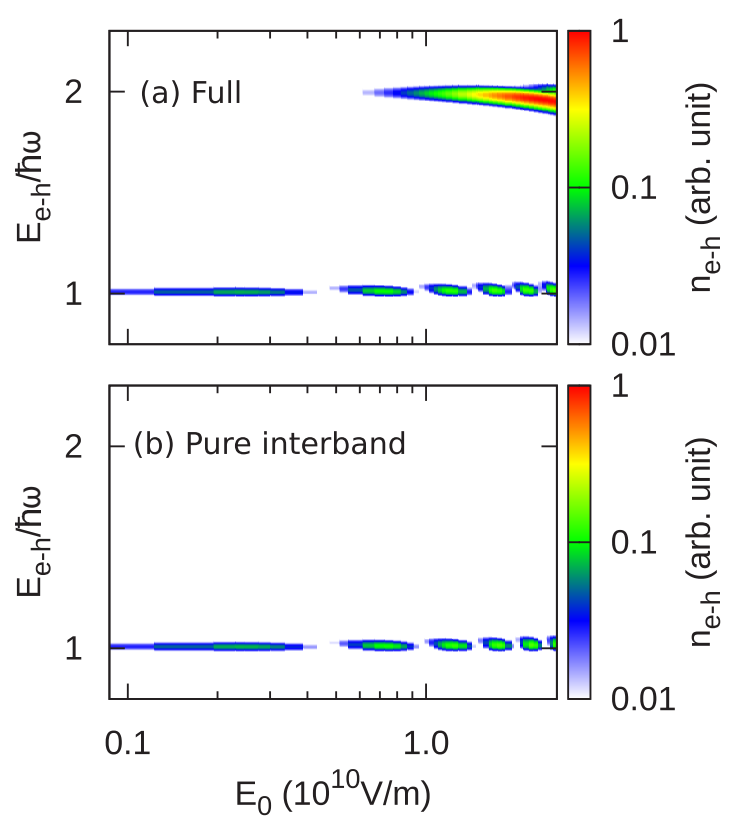

FIG. 8. Distribution of photoinduced electron-hole pairs in the resonant condition. (a) The result of the full model and (b) that of the pure interband model.

two models show different behaviors: While the population of the pure interband model shows oscillations and does not increase much, that of the full model monotonically increases. The oscillating behavior in the pure interband model can be explained by the population and depopulation dynamics of the Rabi flopping. The monotonic increase in the full model means that intraband transitions strongly enhance photocarrier generation and overcome the depopulation mechanism of Rabi flopping.

In order to elucidate the microscopic origin of the enhancement of carrier injection in the resonant condition, we investigate the $e$-h pair distribution after the laser irradiation. Figure 8 shows the $e$-h pair distribution as a function of the $e$-h excitation energy $E_{e-h}$ and the field strength $E_{0}$. Figure 8(a) shows the result of the full model, and Fig. 8(b) shows that of the pure interband model. As seen from Fig. 8(b), the $e$-h pairs are created at around the photon energy of the laser field $\left(E_{e-h} \sim \hbar \omega\right)$ in the entire investigated field strength range. Therefore, photocarrier injection is always dominated by single-photon absorption in the pure interband model. Furthermore, we may see that the population of $e$-h pairs at around the photon energy, $E_{e-h} \sim \hbar \omega$, in the pure interband model shows an oscillating behavior with increasing field strength $E_{0}$. This oscillating behavior corresponds to the oscillations observed in Fig. 7, and it originates from Rabi flopping. In Fig. 8(a), a similar structure can be found at the photon energy of the laser field, $E_{e-h}=\hbar \omega$. This fact indicates that intraband transitions do not directly affect the Rabi flopping dynamics and the single-photon absorption. We see that intraband transitions induce additional large numbers of $e-\mathrm{h}$ pairs at around $E_{e-h}=2 \hbar \omega$. This fact indicates that intraband transitions open a multiphoton excitation channel and strongly enhance the carrier injection once the field strength becomes strong enough.

\section{SUMMARY}

In this work, we investigated the role of intraband transitions in photocarrier generation in semiconductors and insulators based on the parabolic two-band model. The accuracy of the model was demonstrated by comparing it with the nonparabolic Kane's band model and ab initio TDDFT simulations.

We first studied the photocarrier generation under the offresonant condition, where the mean photon energy $\hbar \omega$ is much smaller than the band gap $\epsilon_{g}$. We found that, in this off-resonant regime, intraband transitions are indispensable for the description of photocarrier generation. Furthermore, if intraband transitions are not taken into account, the injection mechanism is dominated by multiphoton absorption even for high laser intensities. This fact can be understood based on the Keldysh parameter $\gamma=\sqrt{\epsilon / 4 U_{p}}$ : the ponderomotive energy $U_{p}$ is induced by the quiver motion in the momentum space, which is an intraband mechanism. Once intraband transitions are ignored, the effective ponderomotive energy vanishes, and the Keldysh parameter becomes infinity. Thus, the excitation mechanism is dominated by the multiphoton process if intraband transitions are ignored. Moreover, we investigated the $e$-h pair distribution as a function of the $e$-h excitation energy. As a result, we found that the $e$-h pair distribution under a strong field shows a multipeak structure with a plateau region (see Fig. 4). A similar feature has been found in ATI photoelectron spectra of atoms. The origin of the plateau of the ATI spectra has been understood as the scattering of ionized electrons from the parent ion based on the semiclassical model [54]. Therefore, the formation of the plateau in the $e$-h pair distribution might also be explained by a semiclassical description.

We then investigated photocarrier generation in the threephoton resonant condition, where the mean photon energy is identical to one third of the band gap. We found that intraband transitions largely enhance the three-photon absorption process. To clarify the origin of the enhancement, we analytically studied the three-photon absorption based on the perturbation theory. As a result, we found that intraband transitions open additional excitation paths that generate photocarriers much more efficiently than the pure interband excitation path. We note that if intraband transitions are induced by a static electric field, the mechanism corresponds to photoassisted tunneling, or the so-called Franz-Keldysh effect [58,59].

We then investigated the carrier-injection in the resonant condition, where the mean photon energy of the laser field is identical to the band gap. When the field is weak enough, the carrier injection is dominated by single-photon absorption, and intraband transitions do not play any role. In contrast, once the field becomes strong enough, intraband transitions significantly enhance the photocarrier generation. Based on the energy-resolved $e$-h distribution analysis, we clarified that the enhancement of the photocarrier generation originates from additional multiphoton excitation paths opened by intraband transitions.

Starting from the above analysis, we can conclude that intraband transitions largely enhance the carrier injection once nonlinear effects become substantial. This finding indicates a potential to control photocarrier injection by employing 
multicolor laser pulses: some of the pulses mainly induce the carrier injection via interband transitions, while the others assist it by opening efficient excitation paths via intraband transitions. Here, in addition to the photon energy of each pulse, the pulse width, the relative time delay, and the relative carrier-envelope phase can be optimizable parameters. Efficient enhancement or suppression of the carrier injection by optical laser pulses will be important for technological applications such as light-driven control of material properties as well as for fundamental investigations of electron dynamics in solids.

\section{ACKNOWLEDGMENTS}

This work was supported by the European Research Council (Grant No. ERC-2015-AdG-694097), Grupos Consolidados (IT578-13), European Union's H2020 program under Grant Agreement No. 676580 (NOMAD), and the National Center of Competence in Research Molecular Ultrafast Science and Technology (NCCR MUST) funded by the Swiss National Science Foundation. S.A.S. gratefully acknowledges the fellowship from the Alexander von Humboldt Foundation.

\section{APPENDIX: PERTURBATION ANALYSIS FOR THREE-PHOTON ABSORPTION PROCESS}

Here, we describe the detailed derivation of Eqs. (18) and (20), analyzing the tree-photon absorption process with the perturbation theory. First, we analyze the pure interband model, ignoring intraband transitions. At the $\Gamma$ point $(\vec{k}=0)$, the Schrödinger equation (5) of the pure interband model can be described as

$$
\begin{gathered}
i \hbar \dot{\mathbf{c}}(t)=H_{\text {inter }}(t) \mathbf{c}(t), \\
H_{\text {inter }}(t)=\left(\begin{array}{cc}
0 & h_{\text {inter }}(t) \\
h_{\text {inter }}^{*}(t) & 0
\end{array}\right),
\end{gathered}
$$

and

$$
h_{\text {inter }}(t)=i \frac{\vec{p}_{v c} \cdot \vec{E}(t)}{\epsilon_{g}} \frac{e \hbar}{m} e^{\frac{1}{i \hbar} \epsilon_{g} t},
$$

where $\mathbf{c}(t)$ is a two-dimensional column vector.

Since $H_{\text {inter }}(t)$ contains only the first-order term of $\vec{E}_{0}$, we can simply consider the following perturbation expansion:

$$
\mathbf{c}(t)=\mathbf{c}^{(0)}(t)+\mathbf{c}^{(1)}(t)+\mathbf{c}^{(2)}(t)+\mathbf{c}^{(3)}(t)+\cdots .
$$

Inserting Eq. (A4) into Eq. (A1), each perturbation order can be evaluated recursively:

$$
\begin{aligned}
& \mathbf{c}^{(1)}(t)=\frac{1}{i \hbar} \int_{0}^{t} d t^{\prime} H_{\text {inter }}\left(t^{\prime}\right) \mathbf{c}^{(0)}\left(t^{\prime}\right), \\
& \mathbf{c}^{(2)}(t)=\frac{1}{i \hbar} \int_{0}^{t} d t^{\prime} H_{\text {inter }}\left(t^{\prime}\right) \mathbf{c}^{(1)}\left(t^{\prime}\right), \\
& \mathbf{c}^{(3)}(t)=\frac{1}{i \hbar} \int_{0}^{t} d t^{\prime} H_{\text {inter }}\left(t^{\prime}\right) \mathbf{c}^{(2)}\left(t^{\prime}\right),
\end{aligned}
$$

and so on.

To simply evaluate the above perturbation expansion, we assume the three-photon resonant condition $\left(3 \hbar \omega=\epsilon_{g}\right)$ and a rectangular envelope with duration $T$ for the applied laser field. Thus, the electric field can be described by

$$
\vec{E}(t)=\vec{E}_{0} \cos (\omega t)
$$

in the domain $0<t<T$ and zero outside of it. Further assuming that the wave function is initially set to the ground state, the third-order coefficient after the laser irradiation can be evaluated as

$$
\begin{aligned}
\mathbf{c}^{(3)}(t>T)= & \frac{1}{(i \hbar)^{3}} \int_{0}^{T} d t^{\prime} \int_{0}^{t^{\prime}} d t^{\prime \prime} \int_{0}^{t^{\prime \prime}} d t^{\prime \prime \prime} H_{\text {inter }}\left(t^{\prime}\right) \\
& \times H_{\text {inter }}\left(t^{\prime \prime}\right) H_{\text {inter }}\left(t^{\prime \prime \prime}\right)\left(\begin{array}{l}
1 \\
0
\end{array}\right)
\end{aligned}
$$

Ignoring oscillatory integrands for $t^{\prime}$, the conduction-band component of $\mathbf{c}^{(3)}(t>T)$ can be simply expressed as

$$
\begin{aligned}
c_{c}^{(3)}(t>T) & =-\frac{1}{8}\left(\frac{\vec{p}_{v c} \cdot \vec{E}_{0}}{\epsilon_{g}} \frac{e \hbar}{m}\right)^{3} \frac{5}{16 \hbar^{3} \omega^{2}} T \\
& =-\frac{1}{8}\left(\frac{\vec{p}_{v c} \cdot \vec{E}_{0}}{\epsilon_{g}} \frac{e \hbar}{m}\right) \frac{15}{16 \hbar^{2} \omega} \Delta_{S} T,
\end{aligned}
$$

where $\Delta_{S}$ is the cycle-averaged Stark shift defined in Eq. (19). In the last line, we employed the three-photon resonant condition, $3 \hbar \omega=\epsilon_{g}$. Finally, the injected population is given by

$$
\begin{aligned}
n_{\Gamma}^{\text {pure-inter }} & =\left|c_{c}(t>T)\right|^{2} \\
& =\left|\frac{1}{8}\left(\frac{\vec{p}_{v c} \cdot \vec{E}_{0}}{\epsilon_{g}} \frac{e \hbar}{m}\right) \frac{15}{16} \frac{1}{\hbar^{2} \omega} \Delta_{S} T\right|^{2} .
\end{aligned}
$$

Note that the first- and the second-order contributions, $\mathbf{c}^{(1)}(t)$ and $\mathbf{c}^{(2)}(t)$, become zero after the laser irradiation in the pure interband model.

Then, we investigate the effect of intraband transitions. Although the Hamiltonian of the pure interband model $H_{\text {inter }}$ contains only the first-order perturbation, that of the full model may contain higher-order perturbations due to intraband transitions. Therefore, intraband transitions open additional excitation paths.

To take intraband transitions into account, we replace the Hamiltonian $H_{\text {inter }}$ by

$$
H(t)=\left(\begin{array}{cc}
0 & h(t) \\
h^{*}(t) & 0
\end{array}\right)
$$

and

$$
h(t)=i \frac{\vec{p}_{v c} \cdot \vec{E}(t)}{\epsilon_{g}} \frac{e \hbar}{m} e^{\frac{1}{i \hbar} \epsilon_{g} t+\frac{1}{i \hbar} \int_{0}^{t} d t^{\prime} \frac{e^{2} A^{2}\left(t^{\prime}\right)}{2 m_{r} c^{2}}} .
$$

Here, the effect of intraband transitions is included via the vector potential $A(t)$. We note that we simply ignored the time dependence of the denominator in $h(t)$. According to Eq. (A8), the vector potential has the following form:

$$
\vec{A}(t)=-c \frac{\vec{E}_{0}}{\omega} \sin (\omega t)
$$

in the domain $0<t<T$ and zero outside of it. 
Assuming a small amplitude for the electric field $\vec{E}_{0}$, the off-diagonal element of Eq. (A13) can be approximated by

$$
h(t)=i \frac{\vec{p}_{v c} \cdot \vec{E}(t)}{\epsilon_{g}} \frac{e \hbar}{m} e^{\frac{1}{i \hbar} \epsilon_{g} t}\left(1+\frac{1}{i \hbar} \int_{0}^{t} d t^{\prime} \frac{e^{2} A^{2}\left(t^{\prime}\right)}{2 m_{r} c^{2}}\right) .
$$

Then, we evaluate Eq. (A5) by replacing $H_{\text {inter }}(t)$ with $H(t)$. Although $\mathbf{c}^{(1)}(t)$ became zero after the laser irradiation in the pure interband model, here, it can be nonzero due to the higherorder contribution in Eq. (A15). Ignoring oscillatory integrands in Eq. (A5), we can easily evaluate the conduction component of $\mathbf{c}^{(1)}(t)$ with $H(t)$ as

$$
c_{c}^{(1)}(t>T)=-\frac{1}{8}\left(\frac{\vec{p}_{v c} \cdot \vec{E}_{0}}{\epsilon_{g}} \frac{e \hbar}{m}\right) \frac{1}{\hbar^{2} \omega} U_{p} T,
$$

and hence,

$$
\begin{aligned}
n_{\Gamma}^{\text {intra-assisted }} & =\left|c_{c}^{(1)}(t>T)\right|^{2} \\
& =\left|\frac{1}{8}\left(\frac{\vec{p}_{v c} \cdot \vec{E}_{0}}{\epsilon_{g}} \frac{e \hbar}{m}\right) \frac{1}{\hbar^{2} \omega} U_{p} T\right|^{2} .
\end{aligned}
$$

[1] F. Krausz and M. Ivanov, Rev. Mod. Phys. 81, 163 (2009).

[2] E. Goulielmakis, Z.-H. Loh, A. Wirth, R. Santra, N. Rohringer, V. S. Yakovlev, S. Zherebtsov, T. Pfeifer, A. M. Azzeer, M. F. Kling, S. R. Leone, and F. Krausz, Nature (London) 466, 739 (2010).

[3] M. Schultze, M. Fieß, N. Karpowicz, J. Gagnon, M. Korbman, M. Hofstetter, S. Neppl, A. L. Cavalieri, Y. Komninos, T. Mercouris, C. A. Nicolaides, R. Pazourek, S. Nagele, J. Feist, J. Burgdörfer, A. M. Azzeer, R. Ernstorfer, R. Kienberger, U. Kleineberg, E. Goulielmakis, F. Krausz, and V. S. Yakovlev, Science 328, 1658 (2010).

[4] E. R. Warrick, W. Cao, D. M. Neumark, and S. R. Leone, J. Phys. Chem. A 120, 3165 (2016).

[5] M. Isinger, R. J. Squibb, D. Busto, S. Zhong, A. Harth, D. Kroon, S. Nandi, C. L. Arnold, M. Miranda, J. M. Dahlström, E. Lindroth, R. Feifel, M. Gisselbrecht, and A. L'Huillier, Science 358, 893 (2017).

[6] M. Schultze, K. Ramasesha, C. Pemmaraju, S. A. Sato, D. Whitmore, A. Gandman, J. S. Prell, L. J. Borja, D. Prendergast, K. Yabana, D. M. Neumark, and S. R. Leone, Science 346, 1348 (2014).

[7] M. Lucchini, S. A. Sato, A. Ludwig, J. Herrmann, M. Volkov, L. Kasmi, Y. Shinohara, K. Yabana, L. Gallmann, and U. Keller, Science 353, 916 (2016).

[8] M. Zürch, H.-T. Chang, L. J. Borja, P. M. Kraus, S. K. Cushing, A. Gandman, C. J. Kaplan, M. H. Oh, J. S. Prell, D. Prendergast, C. D. Pemmaraju, D. M. Neumark, and S. R. Leone, Nat. Commun. 8, 15734 (2017).

[9] S. Ghimire, A. D. DiChiara, E. Sistrunk, P. Agostini, L. F. DiMauro, and D. A. Reis, Nat. Phys. 7, 138 (2011).

[10] G. Vampa, T. Hammond, N. Thiré, B. Schmidt, F. Légaré, C. McDonald, T. Brabec, and P. Corkum, Nature (London) 522, 462 (2015).

[11] T. T. Luu, M. Garg, S. Y. Kruchinin, A. Moulet, M. T. Hassan, and E. Goulielmakis, Nature (London) 521, 498 (2015).

[12] G. Vampa, C. R. McDonald, G. Orlando, D. D. Klug, P. B. Corkum, and T. Brabec, Phys. Rev. Lett. 113, 073901 (2014).

[13] E. N. Osika, A. Chacón, L. Ortmann, N. Suárez, J. A. PérezHernández, B. Szafran, M. F. Ciappina, F. Sols, A. S. Landsman, and M. Lewenstein, Phys. Rev. X 7, 021017 (2017).

[14] K. Uchida, T. Otobe, T. Mochizuki, C. Kim, M. Yoshita, H. Akiyama, L. N. Pfeiffer, K. W. West, K. Tanaka, and H. Hirori, Phys. Rev. Lett. 117, 277402 (2016).
[15] F. Schlaepfer, M. Lucchini, S. A. Sato, M. Volkov, L. Kasmi, N. Hartmann, A. Rubio, L. Gallmann, and U. Keller, Nat. Phys. 14, 560 (2018).

[16] G. Tränkle, H. Leier, A. Forchel, H. Haug, C. Ell, and G. Weimann, Phys. Rev. Lett. 58, 419 (1987).

[17] S. Das Sarma, R. Jalabert, and S.-R. E. Yang, Phys. Rev. B 41, 8288 (1990).

[18] K. Sokolowski-Tinten and D. von der Linde, Phys. Rev. B 61, 2643 (2000).

[19] R. R. Gattass and E. Mazur, Nat. Photonics 2, 219 (2008).

[20] J. Bonse, S. Höhm, S. V. Kirner, A. Rosenfeld, and J. Krüger, IEEE J. Sel. Top. Quantum Electron. 23, 109 (2017).

[21] L. Keldysh, Sov. Phys. JETP 20, 1307 (1965).

[22] V. E. Gruzdev, Phys. Rev. B 75, 205106 (2007).

[23] N. S. Shcheblanov, M. E. Povarnitsyn, P. N. Terekhin, S. Guizard, and A. Couairon, Phys. Rev. A 96, 063410 (2017).

[24] C. R. McDonald, A. B. Taher, and T. Brabec, J. Opt. 19, 114005 (2017).

[25] C. R. McDonald, G. Vampa, P. B. Corkum, and T. Brabec, Phys. Rev. Lett. 118, 173601 (2017).

[26] P. Balling and J. Schou, Rep. Prog. Phys. 76, 036502 (2013).

[27] A.-C. Tien, S. Backus, H. Kapteyn, M. Murnane, and G. Mourou, Phys. Rev. Lett. 82, 3883 (1999).

[28] P. Jürgens, M. Jupé, M. Gyamfi, and D. Ristau, Proc. SPIE 10014, 100141C (2016).

[29] M. S. Wismer, S. Y. Kruchinin, M. Ciappina, M. I. Stockman, and V. S. Yakovlev, Phys. Rev. Lett. 116, 197401 (2016).

[30] Y. Peter and M. Cardona, Fundamentals of Semiconductors: Physics and Materials Properties (Springer, Berlin, 2010).

[31] A. P. Jauho and K. Johnsen, Phys. Rev. Lett. 76, 4576 (1996).

[32] T. Otobe, Y. Shinohara, S. A. Sato, and K. Yabana, Phys. Rev. B 93, 045124 (2016).

[33] E. O. Kane, J. Phys. Chem. Solids 1, 249 (1957).

[34] E. Runge and E. K. U. Gross, Phys. Rev. Lett. 52, 997 (1984).

[35] W. V. Houston, Phys. Rev. 57, 184 (1940).

[36] J. B. Krieger and G. J. Iafrate, Phys. Rev. B 33, 5494 (1986).

[37] M. Cardona and F. H. Pollak, Phys. Rev. 142, 530 (1966).

[38] S. Y. Kruchinin, M. Korbman, and V. S. Yakovlev, Phys. Rev. B 87, 115201 (2013).

[39] E. Kane, J. Phys. Chem. Solids 12, 181 (1960).

[40] N. Troullier and J. L. Martins, Phys. Rev. B 43, 1993 (1991).

[41] A. D. Becke and E. R. Johnson, J. Chem. Phys. 124, 221101 (2006). 
[42] J. P. Perdew and Y. Wang, Phys. Rev. B 45, 13244 (1992).

[43] S. A. Sato and K. Yabana, J. Adv. Simul. Sci. Eng. 1, 98 (2014).

[44] Y. Hirokawa, T. Boku, S. A. Sato, and K. Yabana, Electron Dynamics Simulation with Time-Dependent Density Functional Theory on Large Scale Symmetric Mode Xeon Phi Cluster, IEEE International Parallel and Distributed Processing Symposium Workshops (IPDPSW) (IEEE, Chicago, IL, USA, 2016), pp. 1202-1211.

[45] G. F. Bertsch, J.-I. Iwata, A. Rubio, and K. Yabana, Phys. Rev. B 62, 7998 (2000).

[46] S. A. Sato, Y. Taniguchi, Y. Shinohara, and K. Yabana, J. Chem. Phys. 143, 224116 (2015).

[47] R. C. Weast, Handbook of Physics and Chemistry, 64th ed. (CRC, Boca Raton, FL, 1983).

[48] S. A. Sato, K. Yabana, Y. Shinohara, T. Otobe, K.-M. Lee, and G. F. Bertsch, Phys. Rev. B 92, 205413 (2015).

[49] D. Golde, T. Meier, and S. W. Koch, Phys. Rev. B 77, 075330 (2008).
[50] F. A. Ilkov, J. E. Decker, and S. L. Chin, J. Phys. B 25, 4005 (1992).

[51] P. Agostini, F. Fabre, G. Mainfray, G. Petite, and N. K. Rahman, Phys. Rev. Lett. 42, 1127 (1979).

[52] K. J. Schafer, B. Yang, L. F. DiMauro, and K. C. Kulander, Phys. Rev. Lett. 70, 1599 (1993).

[53] J. Eberly, J. Javanainen, and K. Rza żewski, Phys. Rep. 204, 331 (1991).

[54] G. G. Paulus, W. Becker, W. Nicklich, and H. Walther, J. Phys. B 27, L703 (1994).

[55] P. B. Corkum, Phys. Rev. Lett. 71, 1994 (1993).

[56] M. Lewenstein, P. Balcou, M. Y. Ivanov, A. L'Huillier, and P. B. Corkum, Phys. Rev. A 49, 2117 (1994).

[57] G. Vampa, C. R. McDonald, G. Orlando, P. B. Corkum, and T. Brabec, Phys. Rev. B 91, 064302 (2015).

[58] W. Franz, Z. Naturforsch. A 13, 484 (1958).

[59] L. Keldysh, Sov. Phys. JETP 7, 788 (1958). 